\title{
HAMILTONIAN ELLIPTIC SYSTEMS WITH NONLINEARITIES OF ARBITRARY GROWTH
}

\author{
José A. Cardoso — Jỗo M. do Ó - Everaldo Medeiros
}

ABStRACT. We study the existence of standing wave solutions for the following class of elliptic Hamiltonian-type systems:

$$
\begin{cases}-\hbar^{2} \Delta u+V(x) u=g(v) & \text { in } \mathbb{R}^{N}, \\ -\hbar^{2} \Delta v+V(x) v=f(u) & \text { in } \mathbb{R}^{N},\end{cases}
$$

with $N \geq 2$, where $\hbar$ is a positive parameter and the nonlinearities $f, g$ are superlinear and can have arbitrary growth at infinity. This system is in variational form and the associated energy functional is strongly indefinite. Moreover, in view of unboundedness of the domain $\mathbb{R}^{N}$ and the arbitrary growth of nonlinearities we have lack of compactness. We use a dual variational approach in combination with a mountain-pass type procedure to prove the existence of positive solution for $\hbar$ sufficiently small.

\section{Introduction}

This paper focuses on the existence of positive solutions for the elliptic Hamiltonian system of the form

$$
\begin{cases}-\hbar^{2} \Delta u+V(x) u=g(v) & \text { in } \mathbb{R}^{N}, \\ -\hbar^{2} \Delta v+V(x) v=f(u) & \text { in } \mathbb{R}^{N},\end{cases}
$$

2010 Mathematics Subject Classification. 35J20, 35J25, 35J50.

Key words and phrases. Nonlinear Schrödinger equations; standing waves; variational methods; elliptic systems; nonlinearities of arbitrary growth.

Research partially supported by the National Institute of Science and Technology of Mathematics INCT-Mat, CAPES/MEC/Brazil and CNPq/Brazil. 
where $N \geq 2$ and $\hbar$ is a positive parameter. System (1.1) arises when one looks for standing wave solutions of the system of Schrödinger equations of the form

$$
\left\{\begin{array}{l}
i \hbar \frac{\partial \psi}{\partial t}=-\frac{\hbar^{2}}{2 m} \Delta \psi+W(x) \psi-H_{\varphi}(\psi, \varphi), \quad t \geq 0, x \in \mathbb{R}^{N}, \\
i \hbar \frac{\partial \varphi}{\partial t}=-\frac{\hbar^{2}}{2 m} \Delta \varphi+W(x) \varphi-H_{\psi}(\psi, \varphi), \quad t \geq 0, x \in \mathbb{R}^{N}, \\
\phi(x, t), \psi(x, t) \in \mathbb{C},
\end{array}\right.
$$

which models a lot of phenomena in quantum mechanics. Here $i$ denotes the imaginary unit, $\hbar$ is the Plank constant, $m$ is the particle's mass, $W$ is a continuous potential, and $H: \mathbb{R}^{2} \rightarrow \mathbb{R}$ is an $C^{1}$-function such that

$$
H_{u_{j}}\left(e^{i \theta} u_{1}, e^{i \theta} u_{2}\right)=e^{i \theta} H_{u_{j}}\left(u_{1}, u_{2}\right), \quad \text { for all } u_{j}, \theta \in \mathbb{R},
$$

and $H(u, v)=G(v)+F(u)$, where $F$ and $G$ are the primitives of $f$ and $g$, respectively. Explicitly, we look for solutions of (1.2) in the form

$$
\psi_{1}(t, x)=e^{i(E / \hbar) t} u(x) \quad \text { and } \quad \psi_{2}(t, x)=e^{i(E / \hbar) t} v(x) .
$$

Substituting the ansaz (1.3) into (1.2) and setting $V(x)=W(x)-E \geq 0$ leads to the time-independent coupled nonlinear Schrödinger equations (1.1). Other examples where system (1.1) appears are the study of nonlinear optics and BoseEinstein condensates (see [15]), models of chemotaxis and activation-inhibition, and models of population dynamics (see [18]). Over the last few decades, several authors have studied nonlinear elliptic problems of type (1.1) motivated by applications and richness of methods used to obtain existence and properties of solutions. For the physical motivation we refer to [5], [8], [18], [22], [26] and references therein.

When $u=v$ and $f \equiv g$, the system (1.1) reduces to the scalar equation

$$
-\hbar^{2} \Delta u+V(x) u=f(u) \text { in } \mathbb{R}^{N} .
$$

There are many papers that study equation (1.4) under several assumptions on the potential $V$ and on the growth of the nonlinearity $f$ (see for example [1], [2], [5], [7], [13], [16], [19], [26]). In [19], P. Rabinowitz proved the existence of solution with minimal energy for (1.4), for small $\hbar>0$, when $f$ has subcritical growth and $\liminf _{|x| \rightarrow \infty} V(x)>\inf _{x \in \mathbb{R}^{N}} V(x) \equiv V_{0}>0$. O. Miyagaki [16] studied the critical growth case, namely $f(s)=\lambda|s|^{q-1} s+|s|^{p-1} s$, where $1<q<p \leq$ $(N+2) /(N-2)$, when the potential $V$ is coercive. Another advance in the study of (1.4) was obtained by M. del Pino and P. Felmer [13] assuming $f$ has subcritical growth and $V$ has a strict local minimum in a bounded set $\Lambda \subset \mathbb{R}^{N}$, that is, $\inf _{x \in \bar{\Lambda}} V(x)<\inf _{x \in \partial \Lambda} V(x)$. In fact, they have found a family $u_{\hbar}$ of solutions for equation (1.4) which concentrates around the local minimum of $V$ (see also [7]). Similar results were obtained by C. Alves et al. [2] when $f$ has critical growth. 
In this paper we consider system (1.1) when the potential $V$ is a radial function locally Hölder continuous satisfying:

$\left(\mathrm{V}_{0}\right)$ The set $\mathcal{Z}=\left\{x \in \mathbb{R}^{N}: V(x)=0\right\}$ has nonempty interior.

$\left(\mathrm{V}_{1}\right)$ There is $A>0$ such that the level set $G_{A}=\left\{x \in \mathbb{R}^{N}: V(x)<A\right\}$ has finite Lebesgue measure.

As we have already mentioned, we are looking for positive solutions of (1.1), and so, as usual, we set $f(s)=g(s)=0$ for all $s \leq 0$ and assume:

$\left(\mathrm{H}_{1}\right) f, g \in C^{1}(\mathbb{R})$ and $f(0)=f^{\prime}(0)=0=g(0)=g^{\prime}(0)$;

$\left(\mathrm{H}_{2}\right)$ there exists $\delta^{\prime}>0$ such that

$0<\left(1+\delta^{\prime}\right) f(s) s \leq f^{\prime}(s) s^{2} \quad$ and $\quad 0<\left(1+\delta^{\prime}\right) g(s) s \leq g^{\prime}(s) s^{2}, \quad$ for all $s>0$.

REmark 1.1. Note that condition $\left(\mathrm{H}_{2}\right)$ implies the so-called AmbrosettiRabinowitz condition, namely

$$
\begin{aligned}
& 0<\left(2+\delta^{\prime}\right) F(s):=\int_{0}^{s} f(t) d t \leq f(s) s, \quad \text { for all } s>0 \\
& 0<\left(2+\delta^{\prime}\right) G(s):=\int_{0}^{s} g(t) d t \leq g(s) s, \quad \text { for all } s>0 .
\end{aligned}
$$

We observe that system (1.1) is in the variational form. Taking $H(u, v)=$ $F(u)+G(v)$ is natural to consider the associated functional defined formally by

$$
\mathcal{I}_{\hbar}(u, v)=\int_{\mathbb{R}^{N}}\left(\hbar^{2} \nabla u \nabla v+V(x) u v\right) d x-\int_{\mathbb{R}^{N}} H(u, v) d x,
$$

since (1.1) is the system of Euler-Lagrange equations associated to $\mathcal{I}_{\hbar}$. It should be mentioned that (1.1) is a Hamiltonian system since $H_{u}(u, v)=f(u)$ and $H_{v}(u, v)=g(v)$. This type of systems has been subject of intense study in recent years. We refer the reader to the articles by Ph. Clément et al. [9], J. Hulshof and R. Van der Vorst [14], and B. Sirakov and S.H.M. Soares [25], and see also D. de Figueiredo [10], [11], and references therein for a more recent and complete description of the subject.

The study of system (1.1) by using variational methods presents three main difficulties. First, the apparent lack of compactness in view of unboundedness of the domain $\mathbb{R}^{N}$. This feature has been treated in the scalar case in many papers, see for example, W. Strauss [26], H. Berestycki and P. Lions [5], P. Rabinowitz [19] and M. del Pino and P. Felmer [13]. For elliptic systems, this feature has been studied by D. de Figueiredo and J. Yang [12], and B. Sirakov [23]. Here, we recover the lack of compactness by exploring geometric conditions on the potential $V$ and working in an appropriate subspace of radial symmetric functions $H_{\text {rad }}^{1}\left(\mathbb{R}^{N}\right)$. Second, the arbitrary growth of the nonlinearities $f$ and $g$ prevents the functional (1.6) to be well-defined in the usual Sobolev space. Here, once again, thanks to the behavior of the potential $V$, we can perform a truncation 
argument to obtain an auxiliary system (see Section 2) whose associated functional is well-defined. Third, the associated functional (1.6) is strongly indefinite. To handle this fact, we will apply a dual variational approach used by M. Ramos and J. Yang [21], and by M. Ramos and H. Tavares [20]. See also [4], [6] and references therein. In addition, we refer the reader to A. Ávila and J. Yang [3] for a related dual formulation used for elliptic systems. We also mention that assumptions similar to $\left(\mathrm{V}_{0}\right)-\left(\mathrm{V}_{1}\right)$ were used recently in the papers [1], [8], [17], [24], [25].

1.1. Statement of main results. In our first result we prove the existence of solutions for (1.1) when nonlinearities have polynomial arbitrary growth at infinity.

Theorem 1.2. Assume $\left(\mathrm{V}_{0}\right)-\left(\mathrm{V}_{1}\right)$ and $\left(\mathrm{H}_{1}\right)-\left(\mathrm{H}_{2}\right)$. Furthermore, suppose that

$\left(\mathrm{H}_{3}\right)$ There exist $p, q>2+\delta^{\prime}$ and $l_{1}, l_{2}>0$ such that

$$
\lim _{s \rightarrow+\infty} \frac{f(s)}{s^{p-1}}=l_{1} \quad \text { and } \quad \lim _{s \rightarrow+\infty} \frac{g(s)}{s^{q-1}}=l_{2} .
$$

Then, there exists $\hbar_{0}>0$ such that (1.1) has a weak solution

$$
\left(u_{\hbar}, v_{\hbar}\right) \in H_{\text {rad }}^{1}\left(\mathbb{R}^{N}\right) \times H_{\text {rad }}^{1}\left(\mathbb{R}^{N}\right), \quad \text { for all } \hbar \in\left(0, \hbar_{0}\right] .
$$

Moreover, $u_{\hbar}, v_{\hbar} \in C^{2}\left(\mathbb{R}^{N}\right)$ are positive and $u_{\hbar}(x), v_{\hbar}(x) \rightarrow 0$, as $|x| \rightarrow \infty$.

Next, in complement to the above result, we consider nonlinearities satisfying a kind of global condition but not necessarily polynomial at infinity.

Theorem 1.3. Assume $\left(\mathrm{V}_{0}\right)-\left(\mathrm{V}_{1}\right)$ and $\left(\mathrm{H}_{1}\right)-\left(\mathrm{H}_{2}\right)$. Moreover, suppose that

$\left(\mathrm{H}_{4}\right)$ for all $\varepsilon>0$, there exists $C_{\varepsilon}>0$ such that

$$
f(s) t+g(t) s \leq \varepsilon\left(s^{2}+t^{2}\right)+C_{\varepsilon}(f(s) s+g(t) t), \quad \text { for all } s, t \in \mathbb{R} .
$$

Then there exists $\hbar_{0}>0$ such that (1.1) has a solution

$$
\left(u_{\hbar}, v_{\hbar}\right) \in H_{\mathrm{rad}}^{1}\left(\mathbb{R}^{N}\right) \times H_{\mathrm{rad}}^{1}\left(\mathbb{R}^{N}\right), \quad \text { for all } \hbar \in\left(0, \hbar_{0}\right] .
$$

Furthermore, $u_{\hbar}, v_{\hbar} \in C^{2}\left(\mathbb{R}^{N}\right)$ are positive and $u_{\hbar}(x), v_{\hbar}(x) \rightarrow 0$, as $|x| \rightarrow \infty$.

REMARK 1.4. Note that hypothesis $\left(\mathrm{H}_{3}\right)$ is a growth condition at infinity, but there are no restrictions on the values of $p$ and $q$, that is, it can be allowed in the range subcritical, critical or supercritical. We recall that if $N \geq 3$, the notion of criticality for (1.1) is related with the so-called critical hyperbola

$$
\frac{1}{p}+\frac{1}{q}=1-\frac{2}{N}
$$

where $p, q>1$, see Ph. Clément et al. [9] and J. Hulshof and R. Van der Vorst [14]. 
EXAMPLE 1.5. One can see that nonlinearities

(a) $f(s)=s^{p-1}$ with $p>2+\delta^{\prime}$ and $g(s)=s^{q-1}+s^{r-1}$ with $q \geq r>2+\delta^{\prime}$, satisfy conditions $\left(\mathrm{H}_{1}\right)-\left(\mathrm{H}_{3}\right)$, and satisfy $\left(\mathrm{H}_{4}\right)$ if and only if $p=q$. Furthermore, one can see easily that the pairs of nonlinearities

(b) $f(s)=s^{p-1}$ and $g(s)=s^{2} e^{s}$, with $p>2+\delta^{\prime}$,

(c) $f(s)=s^{2} e^{s}$ and $g(s)=s^{2} e^{s^{2}}$,

(d) $f(s)=s^{3} e^{s^{3}}$ and $g(s)=s^{3} e^{s^{4}}$,

satisfy hypotheses $\left(\mathrm{H}_{1}\right)-\left(\mathrm{H}_{2}\right)$ and $\left(\mathrm{H}_{4}\right)$, however, do not satisfy $\left(\mathrm{H}_{3}\right)$.

This paper is organized as follows. In the next section we modify nonlinearities $f$ and $g$ to get an auxiliary Hamiltonian-type system with the associated energy functional defined in a subspace of $H_{\text {rad }}^{1}\left(\mathbb{R}^{N}\right) \times H_{\text {rad }}^{1}\left(\mathbb{R}^{N}\right)$. In Section 3 , we set up a dual variational formulation in order to obtain a new functional suitable to apply the minimax procedure. Section 4 is devoted to proving the Palais-Smale condition to the energy functional associated to the auxiliary system. Finally, in Section 5, we prove the existence of solutions for the auxiliary system of Section 3 and present the proof of the main results.

\section{Auxiliary elliptic system}

Our approach is based on the minimax method. The natural space for studying weak solutions of system $(1.1)$ is $H_{V}^{1}\left(\mathbb{R}^{N}\right) \times H_{V}^{1}\left(\mathbb{R}^{N}\right)$ where

$$
H_{V}^{1}\left(\mathbb{R}^{N}\right)=\left\{u \in H^{1}\left(\mathbb{R}^{N}\right): \int_{\mathbb{R}^{N}} V(x) u^{2} d x<\infty\right\}
$$

and the associated functional is defined by

$$
\mathcal{I}_{\hbar}(u, v)=\int_{\mathbb{R}^{N}}\left(\hbar^{2} \nabla u \nabla v+V(x) u v\right) d x-\int_{\mathbb{R}^{N}}(F(u)+G(v) d x .
$$

Note that, under assumptions $\left(\mathrm{V}_{0}\right)-\left(\mathrm{V}_{1}\right)$, we have the continuous embedding

$$
H_{V}^{1}\left(\mathbb{R}^{N}\right) \hookrightarrow H^{1}\left(\mathbb{R}^{N}\right) \hookrightarrow L^{r}\left(\mathbb{R}^{N}\right)
$$

for all $2 \leq r \leq 2^{*}$ (see [24] for more details). Then, we see that $\mathcal{I}_{\hbar}$ is well-defined provided that $|F(s)| \leq C|s|^{p}$ and $|G(s)| \leq C|s|^{q}$ with $2 \leq p, q \leq 2^{*}$. As mentioned by Ph. Clément et al. [9], and J. Hulshof and R. Van der Vorst [14], this type of assumptions is too restrictive. To study system (1.1) when nonlinearities have arbitrary growth, one cannot use variational methods based on the functional (2.1) directly, because it is not well-defined in general. To overcome this technical difficulty, inspired by papers [1], [13], [20], we make a convenient modification of nonlinearities $f$ and $g$ so that we can consider a new system that has a associated functional which is well-defined. Explicitly, using $\left(\mathrm{H}_{1}\right)-\left(\mathrm{H}_{2}\right)$, given $\varepsilon>0$, we consider $a_{1}>0$ such that

$$
f^{\prime}\left(a_{1}\right) \leq \varepsilon \quad \text { and } \quad f^{\prime}(s) \geq f^{\prime}\left(a_{1}\right), \quad \text { for all } s \geq a_{1},
$$


and we define the function

$$
\tilde{f}(s)= \begin{cases}f(s) & \text { if } s \leq a_{1}, \\ B_{1} s+\widehat{B}_{1} & \text { if } s \geq a_{1},\end{cases}
$$

where $B_{1}=f^{\prime}\left(a_{1}\right)$ and $\widehat{B}_{1}=f\left(a_{1}\right)-f^{\prime}\left(a_{1}\right) a_{1}$.

REMARK 2.1. Using the assumptions on the potential $V$ we obtain that there exist $0<R_{1}<r_{1}<r_{2}<R_{2}$ and $\alpha>0$ such that

$$
V(x)=0, \quad \text { for all } x \in A_{r_{1}}^{r_{2}}, \quad \text { and } \quad V(x) \geq \alpha>0, \quad \text { for all } x \in \mathbb{R}^{N} \backslash A_{R_{1}}^{R_{2}} \text {, }
$$

where $A_{\rho_{1}}^{\rho_{2}}$ denotes the annulus of radius $0<\rho_{1}<\rho_{2}$, that is,

$$
A_{\rho_{1}}^{\rho_{2}}=\left\{x \in \mathbb{R}^{N}: \rho_{1}<|x|<\rho_{2}\right\} .
$$

Now we consider the auxiliary function $f: \mathbb{R}^{N} \times \mathbb{R} \rightarrow \mathbb{R}$ defined by

$$
f(x, s)= \begin{cases}\chi_{A_{R_{1}}^{R_{2}}} f(s)+\left(1-\chi_{\left.A_{R_{1}}^{R_{2}}\right)}\right) \widetilde{f}(s) & \text { if } s \geq 0, \\ 0 & \text { if } s \leq 0,\end{cases}
$$

where $\chi_{U}$ denotes the characteristic function of the measurable set $U \subset \mathbb{R}^{N}$. Similarly, we can find $a_{2}>0$ and define the corresponding function $g$. The primitives of $f$ and $g$ are respectively, $F(x, s)=\int_{0}^{s} f(x, t) d t$ and $G(x, s)=$ $\int_{0}^{s} g(x, t) d t$.

Next, for an easy reference, we collect some properties of the function $f$ (resp. $g$ ).

Lemma 2.2. The function $f$ is radially symmetric in $x$, that is, $f(|x|, s)=$ $f(|y|, s)$ if $|x|=|y|$. Moreover, $f$ satisfies:

(a) $f(x, s)=f(s)=o(s)$, near the origin, uniformly in $x \in \mathbb{R}^{N}$;

(b) $f(x, s) \leq f(s)$ and $\frac{\partial f}{\partial s}(x, s) \geq 0$ in $\mathbb{R}^{N} \times[0, \infty)$;

(c) (c $\left.c_{1}\right)$ For all $(x, s) \in A_{R_{1}}^{R_{2}} \times(0, \infty) \cup\left(\mathbb{R}^{N} \backslash A_{R_{1}}^{R_{2}}\right) \times\left(-\infty, a_{1}\right]$,

$$
\left(1+\delta^{\prime}\right) f(x, s) s \leq \frac{\partial f}{\partial s}(x, s) s^{2} .
$$

(c $\left.\mathrm{c}_{2}\right)$ For all $(x, s) \in \mathbb{R}^{N} \backslash A_{R_{1}}^{R_{2}} \times(0, \infty)$,

$$
f(x, s) s \leq \frac{\partial f}{\partial s}(x, s) s^{2} .
$$

(c $\left.c_{3}\right)$ Moreover, for all $(x, s) \in \mathbb{R}^{N} \backslash A_{R_{1}}^{R_{2}} \times(0, \infty)$,

$$
2 F(x, s) \leq f(x, s) s \leq \frac{\alpha}{k} s^{2} \leq \frac{1}{k} V(x) s^{2},
$$

where $k \geq 1$ and $\alpha>0$ is given in condition $\left(\mathrm{V}_{1}\right)$.

(d) $F(\cdot, u) \in L^{1}\left(\mathbb{R}^{N}\right)$ provided that $u \in H_{\mathrm{rad}}^{1}\left(\mathbb{R}^{N}\right)$. 
Proof. The proof of items (a)-(c) is an easy consequence of the definition of $f$. To prove $(\mathrm{d})$, if $u \in H_{\mathrm{rad}}^{1}\left(\mathbb{R}^{N}\right)$, by the Strauss lemma (see [26]),

$$
|u(x)| \leq C|x|^{(1-N) / 2}\|u\|_{H^{1}\left(\mathbb{R}^{N}\right)}, \quad \text { for all } x \in \mathbb{R}^{N} \backslash\{0\} .
$$

Therefore,

$$
|u(x)| \leq C\left|R_{1}\right|^{(1-N) / 2}\|u\|_{H^{1}\left(\mathbb{R}^{N}\right)}=c_{1}, \quad \text { for all } x \in A_{R_{1}}^{R_{2}} .
$$

From (2.6), it follows that

$$
\int_{\mathbb{R}^{N}} F(x, u) d x \leq \max _{s \in\left[0, c_{1}\right]} F(s)\left|A_{R_{1}}^{R_{2}}\right|+\int_{\mathbb{R}^{N} \backslash A_{R_{1}}^{R_{2}}} \frac{\alpha}{k} u^{2} d x<\infty,
$$

where $|U|$ denotes the Lebesgue measure of the measurable set $U \subset \mathbb{R}^{N}$.

Now we consider the following auxiliary Hamiltonian system:

$$
\begin{cases}-\hbar^{2} \Delta u+V(x) u=g(x, v) & \text { in } \mathbb{R}^{N}, \\ -\hbar^{2} \Delta v+V(x) v=f(x, u) & \text { in } \mathbb{R}^{N} .\end{cases}
$$

In order to study system (2.8) variationally, we consider the subspace of $H_{\mathrm{rad}}^{1}\left(\mathbb{R}^{N}\right)$

$$
H_{V, \mathrm{rad}}^{1}\left(\mathbb{R}^{N}\right)=\left\{u \in H_{\mathrm{rad}}^{1}\left(\mathbb{R}^{N}\right): \int_{\mathbb{R}^{N}} V(x) u^{2} d x<\infty\right\},
$$

which is a Hilbert space when endowed with the inner product

$$
\langle u, v\rangle=\int_{\mathbb{R}^{N}}[\nabla u \nabla v+V(x) u v] d x, \quad u, v \in H_{V, \mathrm{rad}}^{1}\left(\mathbb{R}^{N}\right),
$$

and its corresponding norm is $\|u\|=\langle u, u\rangle^{1 / 2}$. Note that, under assumptions $\left(\mathrm{V}_{0}\right)-\left(\mathrm{V}_{1}\right)$, we have the continuous embedding

$$
H_{V, \operatorname{rad}}^{1}\left(\mathbb{R}^{N}\right) \hookrightarrow H_{\mathrm{rad}}^{1}\left(\mathbb{R}^{N}\right) \hookrightarrow L^{p}\left(\mathbb{R}^{N}\right),
$$

for all $2 \leq p<\infty$, if $N=2$, and $2 \leq p \leq 2^{*}=2 N /(N-2)$, if $N \geq 3$. We also recall that, due to W. Strauss [26], the last embedding is compact for all $2<p<\infty$, if $N=2$, and $2<p<2^{*}$, if $N \geq 3$. Given $\hbar>0$, we define

$$
\langle u, v\rangle_{\hbar}=\int_{\mathbb{R}^{N}}\left[\hbar^{2} \nabla u \nabla v+V(x) u v\right] d x, \quad u, v \in H_{V, \mathrm{rad}}^{1}\left(\mathbb{R}^{N}\right),
$$

and $E_{\text {rad }}:=H_{V \text {,rad }}^{1}\left(\mathbb{R}^{N}\right) \times H_{V, \text { rad }}^{1}\left(\mathbb{R}^{N}\right)$ endowed with the inner product

$$
\langle(u, v),(\phi, \varphi)\rangle_{\hbar}=\langle u, \phi\rangle_{\hbar}+\langle v, \varphi\rangle_{\hbar}, \quad u, v, \phi, \varphi \in H_{V, \text { rad }}^{1}\left(\mathbb{R}^{N}\right),
$$

and its corresponding norm is $\|(u, v)\|_{\hbar}=\left(\|u\|_{\hbar}^{2}+\|v\|_{\hbar}^{2}\right)^{1 / 2}$.

In view of Lemma 2.2 system (2.8) is variational, and the associated functional $I_{\hbar}: E_{\mathrm{rad}} \rightarrow \mathbb{R}$

$$
I_{\hbar}(u, v)=\langle u, v\rangle_{\hbar}-\int_{\mathbb{R}^{N}}(F(x, u)+G(x, v)) d x
$$


is well-defined. Note that formally the Euler-Lagrange equations associated with $I_{\hbar}$ are precisely the equations in (2.8). Furthermore, $I_{\hbar} \in C^{2}\left(E_{\text {rad }}, \mathbb{R}\right)$ and the first derivative is given by

$$
I_{\hbar}^{\prime}(u, v)(\phi, \varphi)=\langle u, \varphi\rangle_{\hbar}+\langle v, \phi\rangle_{\hbar}-\int_{\mathbb{R}^{N}}[f(x, u) \phi+g(x, v) \varphi] d x,
$$

for all $\phi, \varphi \in H_{V, \text { rad }}^{1}\left(\mathbb{R}^{N}\right)$. The critical points of the functional $I_{\hbar}$ correspond to nonnegative weak solutions of (2.8).

REMARK 2.3. For simplicity, in the next two sections, we will assume that $\hbar=1$ and we will use the following notations: $\langle\cdot, \cdot\rangle=\langle\cdot, \cdot\rangle_{1},\|\cdot\|=\|\cdot\|_{1}$ and $I=I_{1}$.

\section{Reduced functional}

In this section, inspired by the approach of papers [6], [20], [21] allowing to overcome the difficulty that the associated functional $I$ is strongly indefinite, we introduce a reduced functional. Explicitly, fixed $w \in H_{V, \text { rad }}^{1}\left(\mathbb{R}^{N}\right)$, we consider the functional $\mathcal{F}: H_{V, \text { rad }}^{1}\left(\mathbb{R}^{N}\right) \rightarrow \mathbb{R}$ defined by

$$
\mathcal{F}(\psi)=I(w+\psi, w-\psi) .
$$

Proposition 3.1. The functional $\mathcal{F}$ is bounded above and there exists a unique $\psi_{w} \in H_{V, \text { rad }}^{1}\left(\mathbb{R}^{N}\right)$ such that

$$
I\left(w+\psi_{w}, w-\psi_{w}\right)=\sup _{\psi \in H_{V, \text { rad }}^{1}\left(\mathbb{R}^{N}\right)} I(w+\psi, w-\psi) .
$$

Proof. Firstly, we observe that

$$
\begin{aligned}
\mathcal{F}(\psi) & =\|w\|^{2}-\|\psi\|^{2}-\int_{\mathbb{R}^{N}}[F(x, w+\psi)+G(x, w-\psi)] d x, \\
\mathcal{F}^{\prime \prime}(\psi)(\varphi, \phi) & =-2\langle\varphi, \phi\rangle-\int_{\mathbb{R}^{N}}\left[f^{\prime}(x, w+\psi)+g^{\prime}(x, w-\psi)\right] \varphi \phi d x,
\end{aligned}
$$

which together with Lemma 2.2 implies

$$
\mathcal{F}^{\prime \prime}(\psi)(\varphi, \varphi)<0, \quad \text { for all } \psi, \varphi \in H_{V, \text { rad }}^{1}\left(\mathbb{R}^{N}\right), \varphi \neq 0 .
$$

Thus, $\mathcal{F}$ is a strictly concave functional. Furthermore, one can see that $\mathcal{F}$ is weakly upper semi-continuous, so $\mathcal{F}$ achieves the maximum at a single point $\psi \in H_{V, \text { rad }}^{1}\left(\mathbb{R}^{N}\right)$.

Remark 3.2. As a consequence of Proposition 3.1, for each $w \in H_{V, \text { rad }}^{1}\left(\mathbb{R}^{N}\right)$ there is a unique $\psi_{w} \in H_{V, \text { rad }}^{1}\left(\mathbb{R}^{N}\right)$ such that

$$
I^{\prime}\left(w+\psi_{w}, w-\psi_{w}\right)(\phi,-\phi)=0, \quad \text { for all } \phi \in H_{V, \text { rad }}^{1}\left(\mathbb{R}^{N}\right) .
$$

In fact,

$$
I^{\prime}\left(w+\psi_{w}, w-\psi_{w}\right)(\phi,-\phi)=-\mathcal{F}^{\prime}\left(\psi_{w}\right)(\phi), \quad \text { for all } \phi \in H_{V, \text { rad }}^{1}\left(\mathbb{R}^{N}\right) .
$$


Moreover, $\psi_{w}$ is the unique weak solution of the elliptic equation

$$
-2 \Delta \psi+2 V(x) \psi=-f(x, w+\psi)+g(x, w-\psi) \quad \text { in } \mathbb{R}^{N} .
$$

Consequently, the map $\Phi: H_{V \text {,rad }}^{1}\left(\mathbb{R}^{N}\right) \rightarrow H_{V \text {,rad }}^{1}\left(\mathbb{R}^{N}\right), \Phi(w)=\psi_{w}$ is welldefined.

Proposition 3.3. The map $\Phi$ belongs to $C^{1}\left(H_{V, \text { rad }}^{1}\left(\mathbb{R}^{N}\right), H_{V, \text { rad }}^{1}\left(\mathbb{R}^{N}\right)\right)$.

Proof. Let $\left(E_{\mathrm{rad}}\right)^{-}:=\left\{(\psi,-\psi): \psi \in H_{V \text {,rad }}^{1}\left(\mathbb{R}^{N}\right)\right\}$ and consider the maps:

$$
\begin{aligned}
& \tau: E_{\mathrm{rad}} \times\left(E_{\mathrm{rad}}\right)^{-} \rightarrow E_{\mathrm{rad}}, \quad((u, v),(\psi,-\psi)) \mapsto(u, v)+(\psi,-\psi), \\
& I^{\prime}: E_{\mathrm{rad}} \rightarrow\left(E_{\mathrm{rad}}\right)^{\prime} \simeq E_{\mathrm{rad}}, \\
& P: E_{\mathrm{rad}} \rightarrow\left(E_{\mathrm{rad}}\right)^{-}, \quad(u, v) \mapsto\left(\frac{u-v}{2},-\frac{u-v}{2}\right) .
\end{aligned}
$$

Thus, we can define the map $\mathcal{G}: E_{\mathrm{rad}} \times\left(E_{\mathrm{rad}}\right)^{-} \rightarrow\left(E_{\mathrm{rad}}\right)^{-}, \mathcal{G}=P \circ I^{\prime} \circ \tau$. Note that $\mathcal{G}$ is $C^{1}$ and

$$
\begin{aligned}
D_{2} \mathcal{G}((u, v),(\psi,-\psi))(\phi,-\phi) & =P \circ I^{\prime \prime}(\tau((u, v),(\psi,-\psi)))(\phi,-\phi) \\
& =P \circ I^{\prime \prime}((\zeta, \eta))(\phi,-\phi),
\end{aligned}
$$

where $(\zeta, \eta)=(u, v)+(\psi,-\psi)$. Identifying $\left(E_{\mathrm{rad}}\right)^{-}=\left(\left(E_{\mathrm{rad}}\right)^{-}\right)^{\prime}$ and defining $T=P \circ I^{\prime \prime}((\zeta, \eta))$, we obtain

$$
T(\phi,-\phi)(\varphi,-\varphi)=-2\langle\phi, \varphi\rangle-\int_{\mathbb{R}^{N}}\left[f^{\prime}(x, \zeta)+g^{\prime}(x, \eta)\right] \varphi \phi d x,
$$

for all $\varphi, \phi \in H_{V \text {,rad }}^{1}\left(\mathbb{R}^{N}\right)$. We claim that $T$ is one-to-one. In fact, if $T(\phi,-\phi)=$ $(0,0)$ then $T(\phi,-\phi)(\phi,-\phi)=0$, which together with Lemma 2.2 implies

$$
2\|\phi\|^{2}=-\int_{\mathbb{R}^{N}}\left[f^{\prime}(x, \zeta)+g^{\prime}(x, \eta)\right] \phi^{2} d x \leq 0,
$$

and so $\phi=0$. On the other hand, we have

$$
(T+\mathrm{Id})(\phi,-\phi)(\varphi,-\varphi)=-\int_{\mathbb{R}^{N}}\left[f^{\prime}(x, \zeta)+g^{\prime}(x, \eta)\right] \phi \varphi d x,
$$

where $\operatorname{Id}:\left(E_{\mathrm{rad}}\right)^{-} \rightarrow\left(\left(E_{\mathrm{rad}}\right)^{-}\right)^{\prime}, \operatorname{Id}((\phi,-\phi))(\varphi,-\varphi)=2\langle\phi, \varphi\rangle$, for all $\phi, \varphi \in$ $H_{V, \text { rad }}^{1}\left(\mathbb{R}^{N}\right)$. We claim that $(T+\mathrm{Id})$ is a compact operator. For that we write $T+\mathrm{Id}=S_{1}+S_{2}$, where $S_{1}, S_{2}:\left(E_{\mathrm{rad}}\right)^{-} \rightarrow\left(E_{\mathrm{rad}}\right)^{-}=\left(\left(E_{\mathrm{rad}}\right)^{-}\right)^{\prime}$ are defined by

$$
\begin{aligned}
& S_{1}(\phi,-\phi)(\varphi,-\varphi)=\int_{\mathbb{R}^{N}} f^{\prime}(x, \zeta) \phi \varphi d x, \\
& S_{2}(\phi,-\phi)(\varphi,-\varphi)=\int_{\mathbb{R}^{N}} g^{\prime}(x, \eta) \phi \varphi d x .
\end{aligned}
$$

Using the Strauss lemma, we obtain

$$
|\zeta(x)| \leq C\|\zeta\|_{H^{1}\left(\mathbb{R}^{N}\right)}\left|R_{1}\right|^{(1-N) / 2}=: c_{2} \quad \text { in } A_{R_{1}}^{R_{2}} .
$$


We can consider $M_{2}=\max _{s \in\left[0, c_{2}\right]} f^{\prime}(s)$ and $M_{3}=\max _{s \in\left[0, a_{1}\right]} f^{\prime}(s), a_{1}$ defined by (2.3). If $\left(\phi_{n},-\phi_{n}\right) \rightarrow\left(\phi_{0},-\phi_{0}\right)$ in $\left(E_{\text {rad }}\right)^{-}$we have

$$
\int_{\mathbb{R}^{N}} f^{\prime}(x, \zeta)\left|\left(\phi_{n}-\phi_{0}\right) \varphi\right| d x \leq\left(M_{2}+M_{3}+B_{1}\right) \int_{\mathbb{R}^{N}}\left|\left(\phi_{n}-\phi_{0}\right) \varphi\right| d x,
$$

which implies

$$
\left\|S_{1}\left(\phi_{n},-\phi_{n}\right)-S_{1}\left(\phi_{0},-\phi_{0}\right)\right\|_{\left(\left(E_{\mathrm{rad}}\right)^{-}\right)^{\prime}} \rightarrow 0 .
$$

Similarly, we can prove that

$$
\left\|S_{2}\left(\phi_{n},-\phi_{n}\right)-S_{2}\left(\phi_{0},-\phi_{0}\right)\right\|_{\left(\left(E_{\mathrm{rad}}\right)^{-}\right)^{\prime}} \rightarrow 0 .
$$

This proves that $T+\mathrm{Id}$ is a compact operator. From the Fredholm alternative we conclude that $T$ is an isomorphism. Finally, from (3.1), it follows that

$$
\mathcal{G}((w, w),(\Phi(w),-\Phi(w)))=P \circ I^{\prime}((w+\Phi(w), w-\Phi(w)))=0,
$$

for all $w \in H_{V, \text { rad }}^{1}\left(\mathbb{R}^{N}\right)$. Then, by using the Implicit function theorem, we conclude that $\Phi$ is $C^{1}$.

By Proposition 3.1, we can consider the reduced functional $J: H_{V, \text { rad }}^{1}\left(\mathbb{R}^{N}\right) \rightarrow \mathbb{R}$ defined by

$$
\begin{aligned}
J(w) & =I\left(w+\psi_{w}, w-\psi_{w}\right) \\
& =\|w\|^{2}-\left\|\psi_{w}\right\|^{2}-\int_{\mathbb{R}^{N}}\left[F\left(x, w+\psi_{w}\right)+G\left(x, w-\psi_{w}\right)\right] d x .
\end{aligned}
$$

Using the chain rule and (3.1), we obtain

$$
J^{\prime}(w)(\phi)=I^{\prime}\left(w+\psi_{w}, w-\psi_{w}\right)(\phi, \phi), \quad \text { for all } \phi \in H_{V, \text { rad }}^{1}\left(\mathbb{R}^{N}\right) .
$$

Setting $K_{J}:=\left\{w \in H_{V, \text { rad }}^{1}\left(\mathbb{R}^{N}\right): J^{\prime}(w)=0\right\}$ and $K_{I}:=\left\{(u, v) \in E_{\mathrm{rad}}\right.$ : $\left.I^{\prime}(u, v)=0\right\}$, we have the following result (see [6, Proposition 2]).

Proposition 3.4. The map $\mathbf{h}: K_{J} \rightarrow K_{I}$ defined by $\mathbf{h}(w)=\left(w+\psi_{w}, w-\psi_{w}\right)$ is a homeomorphism between critical points of $J$ and critical points of I whose inverse $\mathbf{h}^{-1}: K_{I} \rightarrow K_{J}$ is given by $\mathbf{h}^{-1}(u, v)=(u+v) / 2$.

In view of the previous proposition, we will obtain the existence of solutions for the modified system (2.8) via critical points of the functional $J$. The next result shows that $J$ has the mountain-pass geometry.

Lemma 3.5. Assume $\left(\mathrm{V}_{0}\right)-\left(\mathrm{V}_{1}\right)$ and $\left(\mathrm{H}_{1}\right)-\left(\mathrm{H}_{2}\right)$ hold. Then, the functional $J$ satisfies:

(a) there exist $\beta, \rho>0$ such that $J(w) \geq \beta$ for $\|w\|=\rho$;

(b) there exists $e \in H_{V, \text { rad }}^{1}\left(\mathbb{R}^{N}\right)$, with $\|e\|>\rho$, such that $J(e)<0$. 
Proof. (a) From $\left(\mathrm{H}_{2}\right)$, given $\varepsilon>0$, there exists $\delta>0$ such that, for all $x \in \mathbb{R}^{N}$,

$$
F(x, s) \leq \frac{\varepsilon}{2} s^{2} \quad \text { and } \quad G(x, s) \leq \frac{\varepsilon}{2} s^{2}, \quad \text { for all }|s| \leq \delta .
$$

From (2.7) we can choose $\rho$ sufficiently small such that for $\|w\| \leq \rho$,

$$
\int_{A_{R_{1}}^{R_{2}}} F(x, w) d x \leq \frac{\varepsilon}{2} \int_{A_{R_{1}}^{R_{2}}} w^{2} d x \quad \text { and } \quad \int_{A_{R_{1}}^{R_{2}}} G(x, w) d x \leq \frac{\varepsilon}{2} \int_{A_{R_{1}}^{R_{2}}} w^{2} d x .
$$

Since $J(w)=I\left(w+\psi_{w}, w-\psi_{w}\right) \geq I(w, w)$, by (2.6), we obtain

$$
\begin{aligned}
J(w) & \geq\|w\|^{2}-\int_{\mathbb{R}^{N}}[F(x, w)+G(x, w)] d x \\
& \geq\|w\|^{2}-\varepsilon \int_{A_{R_{1}}^{R_{2}}} w^{2} d x-\frac{1}{k} \int_{\mathbb{R}^{N} \backslash A_{R_{1}}^{R_{2}}} V(x) w^{2} d x \\
& \geq \int_{\mathbb{R}^{N}}\left[|\nabla w|^{2}+\left(1-\frac{1}{k}\right) V(x) w^{2}\right] d x-\varepsilon \int_{A_{R_{1}}^{R_{2}}} w^{2} d x .
\end{aligned}
$$

Now, using that

$$
\int_{A_{R_{1}}^{R_{2}}} w^{2} d x \leq C \int_{\mathbb{R}^{N}}\left[|\nabla w|^{2}+V(x) w^{2}\right] d x
$$

for some $C>0$, we conclude that $J(w) \geq C_{1}\|w\|^{2}$, which proves (a).

In order to prove (b) we consider $\phi \in C_{0}^{\infty}\left(A_{R_{1}}^{R_{2}}\right)$ such that $\phi(x) \geq a_{0}>0$ for all $x \in K$, where $K \subset \operatorname{supp}(\phi)$. For $t \geq 0$, it follows from (1.5) that

$$
\begin{aligned}
J(t \phi) & \leq t^{2}\|\phi\|^{2}-\int_{K} F\left(x, t \phi+\psi_{t \phi}\right) d x-\int_{K} G\left(x, t \phi-\psi_{t \phi}\right) d x \\
& \leq t^{2}\|\phi\|^{2}-C\left(\int_{K}\left(\left(t \phi+\psi_{t \phi}\right)^{+}\right)^{2+\delta^{\prime}} d x+\int_{K}\left(\left(t \phi-\psi_{t \phi}\right)^{+}\right)^{2+\delta^{\prime}} d x\right)+\bar{C},
\end{aligned}
$$

for some constants $C, \bar{C}>0$. Since

$$
(2 t \phi)^{2+\delta^{\prime}} \leq 2^{2+\delta^{\prime}}\left[\left(\left(t \phi+\psi_{t \phi}\right)^{+}\right)^{2+\delta^{\prime}}+\left(\left(t \phi-\psi_{t \phi}\right)^{+}\right)^{2+\delta^{\prime}}\right],
$$

we get

$$
J(t \phi) \leq t^{2}\|\phi\|^{2}-t^{2+\delta^{\prime}} C \int_{K} \phi^{2+\delta^{\prime}} d x+\bar{C},
$$

which implies (b) and this completes the proof.

\section{Palais-Smale condition}

In this section we study the Palais-Smale condition for the functional $I=$ $I_{1}: E_{\mathrm{rad}} \rightarrow \mathbb{R}$ defined in (2.9) (see Remark 2.3). Let $X$ be a Banach space, $I \in C^{1}(X, \mathbb{R})$ and $c \in \mathbb{R}$. We recall that a functional $I$ satisfies the $(\mathrm{PS})_{c}$ condition if any sequence $\left(w_{n}\right)_{n} \subset X$ (called a $(\mathrm{PS})_{c}$ sequence) such that

$$
I\left(w_{n}\right) \rightarrow c \text { and }\left\|I^{\prime}\left(w_{n}\right)\right\|_{X^{\prime}} \rightarrow 0,
$$

has a convergent subsequence. 
REMARK 4.1. One can see that if $I$ satisfies the $(\mathrm{PS})_{c}$ condition then $J$ also satisfies the $(\mathrm{PS})_{c}$ condition.

Lemma 4.2. Assume $\left(\mathrm{V}_{0}\right)-\left(\mathrm{V}_{1}\right)$ and $\left(\mathrm{H}_{1}\right)-\left(\mathrm{H}_{2}\right)$ hold. Under condition $\left(\mathrm{H}_{3}\right)$ or $\left(\mathrm{H}_{4}\right)$ all $(\mathrm{PS})_{c}$ sequences for $I$ are bounded.

Proof. First, assume $\left(\mathrm{H}_{3}\right)$. Invoking (2.10), we get

$$
\left\|u_{n}\right\|^{2}+\left\|v_{n}\right\|^{2}=\int_{\mathbb{R}^{N}}\left[f\left(x, u_{n}\right) v_{n}+g\left(x, v_{n}\right) u_{n}\right] d x+\varepsilon_{n}\left\|\left(u_{n}, v_{n}\right)\right\|,
$$

where $\varepsilon_{n}$ denotes a sequence of positive numbers such that $\varepsilon_{n} \rightarrow 0$ as $n \rightarrow 0$. Since

$$
\begin{aligned}
2 I\left(u_{n}, v_{n}\right)-I^{\prime}\left(u_{n}, v_{n}\right)\left(u_{n}, v_{n}\right)= & \int_{\mathbb{R}^{N}}\left(f\left(x, u_{n}\right) u_{n}-2 F\left(x, u_{n}\right)\right) d x \\
& +\int_{\mathbb{R}^{N}}\left(g\left(x, v_{n}\right) v_{n}-2 G\left(x, v_{n}\right)\right) d x
\end{aligned}
$$

using (2.4)-(2.6), we conclude that

$$
\begin{aligned}
\int_{A_{R_{1}}^{R_{2}}}\left(f\left(x, u_{n}\right) u_{n}+g\left(x, v_{n}\right) v_{n}\right) d x & \leq \frac{2+\delta^{\prime}}{\delta^{\prime}}\left|2 I\left(u_{n}, v_{n}\right)-I^{\prime}\left(u_{n}, v_{n}\right)\left(u_{n}, v_{n}\right)\right| \\
& \leq C+C \varepsilon_{n}\left(\left\|\left(u_{n}, v_{n}\right)\right\|\right) .
\end{aligned}
$$

From $\left(\mathrm{H}_{1}\right)$ and $\left(\mathrm{H}_{3}\right)$, given $\varepsilon>0$, there exist $\delta_{\varepsilon}>0$ and $C_{\varepsilon}>0$ such that

$$
f(s) \leq \varepsilon s, \quad 0 \leq s \leq \delta_{\varepsilon}, \quad \text { and } \quad f(s) \leq C_{\varepsilon} s^{p-1} \quad s \geq \delta_{\varepsilon} .
$$

Now, using (2.7), we have

$$
\begin{aligned}
& \int_{A_{R_{1}}^{R_{2}} \cap\left\{u_{n} \geq \delta_{\varepsilon}\right\}} f\left(u_{n}\right) v_{n} d x \\
& \leq\left(\int_{A_{R_{1}}^{R_{2}} \cap\left\{u_{n} \geq \delta_{\varepsilon}\right\}} f\left(u_{n}\right)^{p /(p-1)} d x\right)^{(p-1) / p}\left(\int_{A_{R_{1}}^{R_{2}}}\left|v_{n}\right|^{p} d x\right)^{1 / p} \\
& \leq C\left\|v_{n}\right\|\left(\int_{A_{R_{1}}^{R_{2}} \cap\left\{u_{n} \geq \delta_{\varepsilon}\right\}} f\left(u_{n}\right) f\left(u_{n}\right)^{1 /(p-1)} d x\right)^{(p-1) / p} \\
& \leq C_{\varepsilon}\left\|v_{n}\right\|\left(\int_{A_{R_{1}}^{R_{2}} \cap\left\{u_{n} \geq \delta_{\varepsilon}\right\}} f\left(u_{n}\right) u_{n} d x\right)^{(p-1) / p}
\end{aligned}
$$

for some $C_{\varepsilon}>0$. Then, we obtain

$$
\begin{aligned}
& \int_{A_{R_{1}}^{R_{2}}} f\left(x, u_{n}\right) v_{n} d x \\
& \quad \leq \varepsilon \int_{A_{R_{1}}^{R_{2}}}\left(u_{n}^{2}+v_{n}^{2}\right) d x+C_{\varepsilon}\left\|\left(u_{n}, v_{n}\right)\right\|\left(C+\varepsilon_{n}\left(\left\|\left(u_{n}, v_{n}\right)\right\|\right)\right)^{(p-1) / p}
\end{aligned}
$$


Similarly, we have

$$
\begin{aligned}
& \int_{A_{R_{1}}^{R_{2}}} g\left(x, v_{n}\right) u_{n} d x \\
& \quad \leq \varepsilon \int_{A_{R_{1}}^{R_{2}}} t\left(u_{n}^{2}+v_{n}^{2}\right) d x+C_{\varepsilon}\left\|\left(u_{n}, v_{n}\right)\right\|\left(C+\varepsilon_{n}\left\|\left(u_{n}, v_{n}\right)\right\|\right)^{(q-1) / q} .
\end{aligned}
$$

From (2.6), we get

$$
\int_{\mathbb{R}^{N} \backslash A_{R_{1}}^{R_{2}}}\left(f\left(x, u_{n}\right) v_{n}+g\left(x, v_{n}\right) u_{n}\right) d x \leq \frac{1}{k} \int_{\mathbb{R}^{N} \backslash A_{R_{1}}^{R_{2}}} V(x)\left(u_{n}^{2}+v_{n}^{2}\right) d x .
$$

Thus, from (4.1)-(4.4), we deduce that

$$
\begin{aligned}
\left\|u_{n}\right\|^{2}+\left\|v_{n}\right\|^{2} \leq & \left(2 C \varepsilon+\frac{1}{k}\right)\left(\left\|u_{n}\right\|^{2}+\left\|v_{n}\right\|^{2}\right)+\left(2 C_{\varepsilon}+\varepsilon_{n}\right)\left\|\left(u_{n}, v_{n}\right)\right\| \\
& +C_{\varepsilon} \varepsilon_{n}\left\|\left(u_{n}, v_{n}\right)\right\|^{(p-1) / p+1}+C_{\varepsilon} \varepsilon_{n}\left\|\left(u_{n}, v_{n}\right)\right\|^{(q-1) / q+1} .
\end{aligned}
$$

Considering $\varepsilon>0$ and $k>1$ such that $(2 C \varepsilon+1 / k)<1$, since $(2 p-1) / p<2$ and $(2 q-1) / q<2$, we conclude that $\left(u_{n}, v_{n}\right)$ is bounded in $E_{\text {rad }}$.

Now assume that $\left(\mathrm{H}_{4}\right)$ holds. Given $\varepsilon>0$, from (4.1) and $\left(\mathrm{H}_{4}\right)$ we have

$$
\begin{aligned}
\left\|u_{n}\right\|^{2}+\left\|v_{n}\right\|^{2} \leq & \varepsilon \int_{A_{R_{1}}^{R_{2}}}\left(u_{n}^{2}+v_{n}^{2}\right) d x+C_{\varepsilon} \int_{A_{R_{1}}^{R_{2}}}\left(f\left(x, u_{n}\right) u_{n}+g\left(x, v_{n}\right) v_{n}\right) d x \\
& +\int_{\mathbb{R}^{N} \backslash A_{R_{1}}^{R_{2}}}\left(f\left(x, u_{n}\right) v_{n}+g\left(x, v_{n}\right) u_{n}\right) d x+\varepsilon_{n}\left\|\left(u_{n}, v_{n}\right)\right\| .
\end{aligned}
$$

Using (4.2) and (4.4), we obtain

$$
\left\|u_{n}\right\|^{2}+\left\|v_{n}\right\|^{2} \leq\left(2 C \varepsilon+\frac{1}{k}\right)\left(\left\|u_{n}\right\|^{2}+\left\|v_{n}\right\|^{2}\right)+C_{\varepsilon}+C_{\varepsilon} \varepsilon_{n}\left\|\left(u_{n}, v_{n}\right)\right\| .
$$

Choosing $\varepsilon>0$ and $k>1$ such that $(2 C \varepsilon+1 / k)<1$, we conclude that $\left(u_{n}, v_{n}\right)$ is bounded in $E_{\mathrm{rad}}$.

Following the paper of M. del Pino and P. Felmer [13], in order to obtain the Palais-Smale condition, the next result plays an important role.

Lemma 4.3. Assume $\left(\mathrm{V}_{0}\right)-\left(\mathrm{V}_{1}\right)$ and $\left(\mathrm{H}_{1}\right)-\left(\mathrm{H}_{2}\right)$ hold. Let $\left(u_{n}, v_{n}\right)_{n} \subset E_{\mathrm{rad}}$ be a bounded sequence such that

$$
\left\|I^{\prime}\left(u_{n}, v_{n}\right)\right\|_{\left(E_{\mathrm{rad}}\right)^{\prime}} \rightarrow 0, \quad n \rightarrow \infty .
$$

Then, given $\varepsilon>0$, there exists $R_{0}>0$ such that for all $R>R_{0}$ it holds

$$
\left.\limsup _{n \rightarrow \infty} \int_{\{|x|>R\}}\left[\left|\nabla u_{n}\right|^{2}+V(x) u_{n}^{2}\right] d x<\varepsilon \quad \text { (respectively, } v_{n}\right) .
$$


Proof. Let $R>4 R_{2}\left(R_{2}\right.$ from hypotheses $\left.\left(\mathrm{V}_{1}\right)\right)$, consider a cut-off function $\eta_{R} \in C_{0}^{\infty}\left(\mathbb{R}^{N},[0,1]\right)$ defined by

$$
\eta_{R}(x)= \begin{cases}0 & \text { if }|x| \leq R / 2 \\ 1 & \text { if }|x| \geq R\end{cases}
$$

and $\left|\nabla \eta_{R}\right| \leq c / R$, for some $c>0$. Here we will use the following notation:

$$
\left.\left\|u_{n}\right\|_{\eta_{R}}^{2}=\int_{\mathbb{R}^{N}}\left[\left|\nabla u_{n}\right|^{2}+V(x) u_{n}^{2}\right] \eta_{R} d x \quad \text { (respectively, } v_{n}\right) .
$$

Since $\left(u_{n}, v_{n}\right)$ is bounded in $E_{\text {rad }}$, we have $I^{\prime}\left(u_{n}, v_{n}\right)\left(\eta_{R} v_{n}, \eta_{R} u_{n}\right)=\mathrm{o}_{n}(1)$, that is,

$$
\begin{aligned}
\left\|u_{n}\right\|_{\eta_{R}}^{2}+\left\|v_{n}\right\|_{\eta_{R}}^{2}= & \int_{\mathbb{R}^{N}}\left(f\left(x, u_{n}\right) v_{n}+g\left(x, v_{n}\right) u_{n}\right) \eta_{R} d x \\
& \quad-\int_{\mathbb{R}^{N}} u_{n} \nabla u_{n} \cdot \nabla \eta_{R}-\int_{\mathbb{R}^{N}} v_{n} \nabla v_{n} \cdot \nabla \eta_{R}+\mathrm{o}_{n}(1) .
\end{aligned}
$$

From (2.6), we conclude that

$$
\int_{\mathbb{R}^{N}}\left(f\left(x, u_{n}\right) v_{n}+g\left(x, v_{n}\right) u_{n}\right) \eta_{R} d x \leq \frac{1}{k}\left(\left\|u_{n}\right\|_{\eta_{R}}^{2}+\left\|v_{n}\right\|_{\eta_{R}}^{2}\right) .
$$

Then, by (4.5), we obtain

$$
\left(1-\frac{1}{k}\right)\left(\left\|u_{n}\right\|_{\eta_{R}}^{2}+\left\|v_{n}\right\|_{\eta_{R}}^{2}\right) \leq \frac{c}{R}\left(\left\|u_{n}\right\|_{L^{2}}\left\|\nabla u_{n}\right\|_{L^{2}}+\left\|v_{n}\right\|_{L^{2}}\left\|\nabla v_{n}\right\|_{L^{2}}\right)+\mathrm{o}_{n}(1) .
$$

Since $\left(u_{n}, v_{n}\right)$ is bounded, choosing $R$ sufficiently large we obtain the desired result.

REMARK 4.4. One can see that if $\left(u_{n}, v_{n}\right)_{n} \rightarrow(u, v)$ weakly in $E_{\text {rad }}$ so that $\left\|I^{\prime}\left(u_{n}, v_{n}\right)\right\|=\mathrm{o}_{n}(1)$ then $(u, v)$ is a critical point of $I$.

Lemma 4.5. Assume $\left(\mathrm{V}_{0}\right)-\left(\mathrm{V}_{1}\right),\left(\mathrm{H}_{1}\right)-\left(\mathrm{H}_{2}\right)$ and $\left(\mathrm{H}_{3}\right)\left(\right.$ or $\left.\left(\mathrm{H}_{4}\right)\right)$ hold. Then the functional I satisfies the $(\mathrm{PS})_{c}$ condition for all $c \in \mathbb{R}$.

Proof. Let $\left(u_{n}, v_{n}\right) \subset E_{\text {rad }}$ be a $(\mathrm{PS})_{c}$ sequence for the functional $I$. By Lemma 4.2 , we have $\left\|\left(u_{n}, v_{n}\right)\right\| \leq C$. Then, up to a subsequence,

$$
u_{n} \rightarrow u \quad \text { and } \quad v_{n} \rightarrow v \quad \text { in } H_{V, \operatorname{rad}}^{1}\left(\mathbb{R}^{N}\right) .
$$

Since $I^{\prime}\left(u_{n}, v_{n}\right)\left(v_{n}, u_{n}\right)=\mathrm{o}_{n}(1)=I^{\prime}\left(u_{n}, v_{n}\right)(v, u)$ and $I^{\prime}(u, v)\left(v_{n}, u_{n}\right)=0=$ $I^{\prime}(u, v)(v, u)$, we obtain

$$
\begin{aligned}
&\| 4.6) \quad\left\|u_{n}-u\right\|^{2}+\left\|v_{n}-v\right\|^{2}=\int_{\mathbb{R}^{N}}\left(f\left(x, u_{n}\right)-f(x, u)\right) v_{n} d x \\
&+\int_{\mathbb{R}^{N}}\left(g\left(x, v_{n}\right)-g(x, v)\right) u_{n} d x+\int_{\mathbb{R}^{N}}\left(f(x, u)-f\left(x, u_{n}\right)\right) v d x \\
&+\int_{\mathbb{R}^{N}}\left(g(x, v)-g\left(x, v_{n}\right)\right) u d x+\mathrm{o}_{n}(1) .
\end{aligned}
$$


We claim that

$$
\int_{\mathbb{R}^{N}}\left(f\left(x, u_{n}\right)-f(x, u)\right) v_{n} d x \rightarrow 0, \quad n \rightarrow \infty .
$$

In fact, we consider a ball $B_{R}=B_{R}(0)$ with radius sufficiently large such that $A_{R_{1}}^{R_{2}} \subset B_{R}$. Then, using (2.6) and Lemma 4.3, there exists $R>0$ sufficiently large such that

$$
\int_{\mathbb{R}^{N} \backslash B_{R}}\left(f\left(x, u_{n}\right)-f(x, u)\right) v_{n} d x=\mathrm{o}_{n}(1) .
$$

The Sobolev embedding together with (2.6) implies

$$
\int_{B_{R} \backslash A_{R_{1}}^{R_{2}}}\left(f\left(x, u_{n}\right)-f(x, u)\right)^{2} d x \rightarrow 0, \quad n \rightarrow \infty .
$$

Since $\left(v_{n}\right)$ is bounded in $L^{2}\left(\mathbb{R}^{N}\right)$, we obtain

$$
\int_{B_{R} \backslash A_{R_{1}}^{R_{2}}}\left(f\left(x, u_{n}\right)-f(x, u)\right) v_{n} d x=\mathrm{o}_{n}(1) .
$$

Moreover, using (2.7) and Lemma 4.2, we have $\left|u_{n}(x)\right| \leq C_{1}$ and $\left|v_{n}(x)\right| \leq C_{2}$, for almost every $x \in A_{R_{1}}^{R_{2}}$, for some constants $C_{1}, C_{2}>0$, and we conclude that

$$
\int_{A_{R_{1}}^{R_{2}}}\left(f\left(x, u_{n}\right)-f(x, u)\right) v_{n} d x=\mathrm{o}_{n}(1) .
$$

Hence, by (4.7)-(4.9), the claim is proved. Finally, an analogous claim with similar terms of (4.6) holds. Therefore, the proof of the lemma is complete.

\section{Proof of Theorems 1.2 and 1.3}

Firstly we will prove the existence of solution for the modified system (2.8). In fact we will show that this solution is also a solution of (1.1). For each $\hbar>0$ and $w \in H_{V \text {,rad }}^{1}\left(\mathbb{R}^{N}\right)$, we get

$$
\begin{aligned}
J_{\hbar}(w) & =I_{\hbar}\left(w+\psi_{w}, w-\psi_{w}\right) \\
& =\|w\|_{\hbar}^{2}-\left\|\psi_{w}\right\|_{\hbar}^{2}-\int_{\mathbb{R}^{N}}\left[F\left(x, w+\psi_{w}\right)+G\left(x, w-\psi_{w}\right)\right] d x .
\end{aligned}
$$

Moreover, for all $\varphi \in H_{V \text {,rad }}^{1}\left(\mathbb{R}^{N}\right)$, we have

$$
\begin{aligned}
J_{\hbar}^{\prime}(w)(\varphi) & =I_{\hbar}^{\prime}\left(w+\psi_{w}, w-\psi_{w}\right)(\varphi, \varphi) \\
& =2\langle w, \varphi\rangle_{\hbar}-\int_{\mathbb{R}^{N}}\left[f\left(x, w+\psi_{w}\right)+g\left(x, w-\psi_{w}\right)\right] \varphi d x .
\end{aligned}
$$

Proposition 5.1. Assume $\left(\mathrm{V}_{0}\right)-\left(\mathrm{V}_{1}\right),\left(\mathrm{H}_{1}\right)-\left(\mathrm{H}_{2}\right)$ and $\left(\mathrm{H}_{3}\right)$ (or $\left.\left(\mathrm{H}_{4}\right)\right)$ hold. Then, for each $\hbar>0$, the functional $J_{\hbar}$ possesses a nontrivial critical point $w_{\hbar} \in H_{\mathrm{rad}}^{1}\left(\mathbb{R}^{N}\right)$ such that

$$
J_{\hbar}\left(w_{\hbar}\right)=c_{\hbar}=\inf _{\gamma \in \Gamma} \max _{t \in[0,1]} J_{\hbar}(\gamma(t))>0,
$$


where $\Gamma=\left\{\gamma \in C\left([0,1], H_{V, \text { rad }}^{1}\left(\mathbb{R}^{N}\right)\right): \gamma(0)=0\right.$ and $\left.J_{\hbar}(\gamma(1))<0\right\}$. Moreover, $\left(u_{\hbar}, v_{\hbar}\right):=\left(w_{\hbar}+\psi_{w_{\hbar}}, w_{\hbar}-\psi_{w_{\hbar}}\right)$ is a $C^{2}\left(\mathbb{R}^{N}\right)$ positive solution of $(2.8)$ such that

$$
I_{\hbar}\left(u_{\hbar}, v_{\hbar}\right)=c_{\hbar} .
$$

Proof. Invoking Lemmas 3.5, 4.5 and the mountain-pass theorem, we obtain a critical point $w_{\hbar}$ of $J_{\hbar}$. In view of Proposition 3.4 we obtain that $\left(w_{\hbar}+\psi_{w_{\hbar}}, w_{\hbar}-\right.$ $\left.\psi_{w_{\hbar}}\right)$ is a critical point of $I_{\hbar}$. Denoting $u_{\hbar}=w_{\hbar}+\psi_{w_{\hbar}}$ and $v_{\hbar}=w_{\hbar}-\psi_{w_{\hbar}}$ and considering $u_{\hbar}^{-}=\min \left\{0, u_{\hbar}\right\}$, we have $I_{\hbar}^{\prime}\left(u_{\hbar}, v_{\hbar}\right)\left(0, u_{\hbar}^{-}\right)=0$, that is,

$$
-\int_{\mathbb{R}^{N}}\left(\hbar^{2}\left|\nabla u_{\hbar}^{-}\right|^{2}+V(x)\left(u_{\hbar}^{-}\right)^{2}\right) d x=\int_{\mathbb{R}^{N}} g\left(x, v_{\hbar}\right) u_{\hbar}^{-} d x \geq 0 .
$$

Therefore, $u_{\hbar} \geq 0$. Similarly, we obtain $v_{\hbar} \geq 0$ and, using the maximum principle, we conclude that $u_{\hbar}$ and $v_{\hbar}$ are positive.

REMARK 5.2. Using standard elliptic regularity, one can see that $w_{\hbar}, \psi_{w_{\hbar}} \in$ $C^{2}\left(\mathbb{R}^{N}\right), u_{\hbar}=w_{\hbar}+\psi_{w_{\hbar}}$ and $v_{\hbar}=w_{\hbar}-\psi_{w_{\hbar}}$ are positive. Indeed, adding the equations of (1.1) we obtain

$$
-\hbar^{2} \Delta w_{\hbar}+V(x) w_{\hbar}=f\left(x, u_{\hbar}\right)+g\left(x, v_{\hbar}\right) \quad \text { in } \mathbb{R}^{N} .
$$

Then, we can apply classical regularity theory to equations (3.2) and (5.2) using properties of $f$ and $g$.

Lemma 5.3. The minimax level $c_{\hbar}$ defined in (5.1) satisfies

$$
c_{\hbar}=\mathrm{o}\left(\hbar^{2}\right), \quad \text { as } \hbar \rightarrow 0 .
$$

Proof. Consider $\phi \in C_{0, \text { rad }}^{\infty}\left(A_{r_{1}}^{r_{2}}\right)$ such that $\phi(x) \geq 0$, for all $x \in \mathbb{R}^{N}$, and $\phi(x) \geq a_{0}>0$, for all $x \in K$, where $K \subset \operatorname{supp}(\phi)$ has positive measure. Following the same arguments as in the proof of Lemma 3.5, we obtain $J_{\hbar}(t \phi) \rightarrow$ $-\infty$ as $t \rightarrow \infty$. Thus,

$$
c_{\hbar} \leq \max _{t \geq 0} J_{\hbar}(t \phi)=J_{\hbar}\left(t_{\hbar} \phi\right),
$$

for some $t_{\hbar}>0$. Since $V(x)=0$, for all $x \in A_{r_{1}}^{r_{2}}$, we deduce that

$$
c_{\hbar} \leq J_{\hbar}\left(t_{\hbar} \phi\right) \leq t_{\hbar}^{2} \int_{A_{r_{1}}^{r_{2}}} \hbar^{2}|\nabla \phi|^{2} d x .
$$

On the other hand, from $J_{\hbar}^{\prime}\left(t_{\hbar} \phi\right)(\phi)=0$, we have

$$
\begin{aligned}
t_{\hbar} \int_{A_{r_{1}}^{r_{2}}} \hbar^{2}|\nabla \phi|^{2} d x & =\int_{\mathbb{R}^{N}}\left[f\left(x, t_{\hbar} \phi+\psi_{t_{\hbar} \phi}\right)+g\left(x, t_{\hbar} \phi-\psi_{t_{\hbar} \phi}\right)\right] \phi d x \\
& \geq \int_{K}\left[f\left(t_{\hbar} \phi+\psi_{t_{\hbar} \phi}\right)+g\left(t_{\hbar} \phi-\psi_{t_{\hbar} \phi}\right)\right] \phi d x .
\end{aligned}
$$


Now observe that either there exists $K_{1} \subset K$, with positive measure, such that $\psi_{t_{\hbar} \phi} \geq 0$ in $K_{1}$, or there exists $K_{2} \subset K$, also with positive measure, such that $\psi_{t_{\hbar} \phi} \leq 0$ in $K_{2}$. In the first case, since $f(s)$ is increasing for $s>0$, we obtain $t_{\hbar} \int_{A_{r_{1}}^{r_{2}}} \hbar^{2}|\nabla \phi|^{2} d x \geq \int_{K_{1}} f\left(t_{\hbar} \phi+\psi_{t_{\hbar} \phi}\right) \phi d x \geq \int_{K_{1}} f\left(t_{\hbar} \phi\right) \phi d x \geq a_{0}^{2} \int_{K_{1}} \frac{f\left(t_{\hbar} \phi\right)}{\phi} d x$. Now, since $f(s) / s$ also is increasing and $\lim _{s \rightarrow \infty} f(s) / s=\infty$, we conclude that $t_{\hbar} \rightarrow 0$ as $\hbar \rightarrow 0$. On the other hand, if $\psi_{t_{\hbar} \phi} \leq 0$ in $K_{2}$ then $-\psi_{t_{\hbar} \phi} \geq 0$ in $K_{2}$. Since $g(s)$ is increasing, by (5.4), we have that

$$
t_{\hbar} \int_{A_{r_{1}}^{r_{2}}} \hbar^{2}|\nabla \phi|^{2} d x \geq \int_{K_{2}} g\left(t_{\hbar} \phi-\psi_{t_{\hbar} \phi}\right) \phi d x \geq a_{0}^{2} \int_{K_{2}} \frac{g\left(t_{\hbar} \phi\right)}{\phi} d x .
$$

Thus, we obtain that $t_{\hbar} \rightarrow 0$ as $\hbar \rightarrow 0$. Therefore, by (5.3), the proof is complete.

Lemma 5.4. Assume $\left(\mathrm{V}_{0}\right)-\left(\mathrm{V}_{1}\right)$ and $\left(\mathrm{H}_{1}\right)-\left(\mathrm{H}_{3}\right)$ hold. If $\left(u_{\hbar}, v_{\hbar}\right)=\left(w_{\hbar}+\psi_{w_{\hbar}}\right.$, $\left.w_{\hbar}-\psi_{w_{\hbar}}\right)$ is a solution of (2.8), obtained in Proposition 5.1, then

$$
\left\|\left(u_{\hbar}, v_{\hbar}\right)\right\|_{H^{1} \times H^{1}}=\mathrm{o}\left(\hbar^{r}\right), \quad \text { as } \hbar \rightarrow 0,
$$

where $r=\min \{(p-2) / p,(q-2) / q\}>0$.

Proof. Note that

$$
\left\|u_{\hbar}\right\|_{\hbar}^{2}+\left\|v_{\hbar}\right\|_{\hbar}^{2}=\int_{\mathbb{R}^{N}}\left(f\left(x, u_{\hbar}\right) v_{\hbar}+g\left(x, v_{\hbar}\right) u_{\hbar}\right) d x
$$

and

$$
\begin{aligned}
2 I_{\hbar}\left(u_{\hbar}, v_{\hbar}\right)-I_{\hbar}^{\prime}\left(u_{\hbar}, v_{\hbar}\right)\left(u_{\hbar}, v_{\hbar}\right)= & \int_{\mathbb{R}^{N}}\left(f\left(x, u_{\hbar}\right) u_{\hbar}-2 F\left(x, u_{\hbar}\right)\right) d x \\
& +\int_{\mathbb{R}^{N}}\left(g\left(x, v_{\hbar}\right) v_{\hbar}-2 G\left(x, v_{\hbar}\right)\right) d x .
\end{aligned}
$$

From (2.6), we obtain

$$
\int_{A_{R_{1}}^{R_{2}}}\left(f\left(x, u_{\hbar}\right) u_{\hbar}-2 F\left(x, u_{\hbar}\right)\right) d x+\int_{A_{R_{1}}^{R_{2}}}\left(g\left(x, v_{\hbar}\right) v_{\hbar}-2 G\left(x, v_{\hbar}\right)\right) d x \leq 2 c_{\hbar} .
$$

By (2.4)-(2.6), we deduce that

$$
\int_{A_{R_{1}}^{R_{2}}}\left(f\left(x, u_{\hbar}\right) u_{\hbar}+g\left(x, v_{\hbar}\right) v_{\hbar}\right) d x \leq \frac{2+\delta^{\prime}}{\delta^{\prime}} 2 c_{\hbar} .
$$

Now, analogously to (4.2), we have

$$
\int_{A_{R_{1}}^{R_{2}}} f\left(x, u_{\hbar}\right) v_{\hbar} d x \leq \varepsilon \int_{A_{R_{1}}^{R_{2}}}\left(u_{\hbar}^{2}+v_{\hbar}^{2}\right) d x+C c_{\hbar}^{(p-1) / p}\left\|\left(u_{\hbar}, v_{\hbar}\right)\right\|_{\hbar},
$$

and

$$
\int_{A_{R_{1}}^{R_{2}}} g\left(x, v_{\hbar}\right) u_{\hbar} d x \leq \varepsilon \int_{A_{R_{1}}^{R_{2}}}\left(u_{\hbar}^{2}+v_{\hbar}^{2}\right) d x+C c_{\hbar}^{(q-1) / q}\left\|\left(u_{\hbar}, v_{\hbar}\right)\right\|_{\hbar}
$$


where $C=C(\varepsilon)>0$. Then, using (2.6), (5.5) and (5.7)-(5.8), we conclude that

$$
\begin{aligned}
\left\|u_{\hbar}\right\|_{\hbar}^{2}+\left\|v_{\hbar}\right\|_{\hbar}^{2} \leq\left(2 C \varepsilon+\frac{1}{k}\right)\left(\left\|u_{\hbar}\right\|_{\hbar}^{2}\right. & \left.+\left\|v_{\hbar}\right\|_{\hbar}^{2}\right) \\
& +2 C_{\varepsilon}\left\|\left(u_{\hbar}, v_{\hbar}\right)\right\|_{\hbar}\left(c_{\hbar}^{(p-1) / p}+c_{\hbar}^{(q-1) / q}\right) .
\end{aligned}
$$

Considering $\varepsilon>0$ and $k>1$ such that $(2 C \varepsilon+1 / k)<1 / 2$, we obtain

$$
\hbar\left\|\left(u_{\hbar}, v_{\hbar}\right)\right\|_{H^{1} \times H^{1}} \leq C_{1}\left\|\left(u_{\hbar}, v_{\hbar}\right)\right\|_{\hbar} \leq 4 \widetilde{C}\left(c_{\hbar}^{(p-1) / p}+c_{\hbar}^{(q-1) / q}\right) .
$$

Applying Lemma 5.3, we obtain the desired result.

Lemma 5.5. Assume $\left(\mathrm{V}_{0}\right)-\left(\mathrm{V}_{1}\right),\left(\mathrm{H}_{1}\right)-\left(\mathrm{H}_{2}\right)$ and $\left(\mathrm{H}_{4}\right)$ hold. If $\left(u_{\hbar}, v_{\hbar}\right)=$ $\left(w_{\hbar}+\psi_{w_{\hbar}}, w_{\hbar}-\psi_{w_{\hbar}}\right)$ is a solution of (2.8), obtained in Proposition 5.1, then

$$
\left\|\left(u_{\hbar}, v_{\hbar}\right)\right\|_{H^{1} \times H^{1}}=\mathrm{o}_{\hbar}(1), \quad \text { as } \hbar \rightarrow 0 .
$$

Proof. From (5.5) and $\left(\mathrm{H}_{4}\right)$, given $\varepsilon>0$, there exists $C_{\varepsilon}>0$ such that

$$
\begin{gathered}
\left\|u_{\hbar}\right\|_{\hbar}^{2}+\left\|v_{\hbar}\right\|_{\hbar}^{2} \leq \varepsilon \int_{A_{R_{1}}^{R_{2}}}\left(u_{\hbar}^{2}+v_{\hbar}^{2}\right) d x+C_{\varepsilon} \int_{A_{R_{1}}^{R_{2}}}\left(f\left(x, u_{\hbar}\right) u_{\hbar}+g\left(x, v_{\hbar}\right) v_{\hbar}\right) d x \\
\int_{\mathbb{R}^{N} \backslash A_{R_{1}}^{R_{2}}}\left(f\left(x, u_{\hbar}\right) v_{\hbar}+g\left(x, v_{\hbar}\right) u_{\hbar}\right) d x .
\end{gathered}
$$

Using (2.6) and (5.6), we get

$$
\left\|u_{\hbar}\right\|_{\hbar}^{2}+\left\|v_{\hbar}\right\|_{\hbar}^{2} \leq\left(C \varepsilon+\frac{1}{k}\right)\left(\left\|u_{\hbar}\right\|_{\hbar}^{2}+\left\|v_{\hbar}\right\|_{\hbar}^{2}\right)+2 C_{\varepsilon} \frac{2+\delta^{\prime}}{\delta^{\prime}} c_{\hbar} .
$$

Now, choosing $\varepsilon>0$ and $k>1$ such that $(C \varepsilon+1 / k)<1 / 2$ and assuming that $\hbar \leq 1$, we obtain

$$
\frac{\hbar^{2}}{2}\left(\left\|u_{\hbar}\right\|_{H^{1}}^{2}+\left\|v_{\hbar}\right\|_{H^{1}}^{2}\right) \leq \frac{1}{2}\left(\left\|u_{\hbar}\right\|_{\hbar}^{2}+\left\|v_{\hbar}\right\|_{\hbar}^{2}\right) \leq \tilde{C} c_{\hbar},
$$

where $\widetilde{C}>0$ is independent of $\hbar$. Applying Lemma 5.3, we complete the proof. $\square$

Proof of Theorem 1.2. For each $\hbar>0$, we consider $\left(u_{\hbar}, v_{\hbar}\right)$ a solution of (2.8), obtained in Proposition 5.1. By (2.7), we have

$$
u_{\hbar}(x) \leq \frac{C\left\|u_{\hbar}\right\|_{H^{1}}}{|x|^{(N-1) / 2}} \quad \text { and } \quad v_{\hbar}(x) \leq \frac{C\left\|v_{\hbar}\right\|_{H^{1}}}{|x|^{(N-1) / 2}}
$$

almost everywhere in $\mathbb{R}^{N} \backslash\{0\}$. We denote

$$
m_{\hbar}=\max \left\{\max _{x \in \partial A_{R_{1}}^{R_{2}}} u_{\hbar}(x), \max _{x \in \partial A_{R_{1}}^{R_{2}}} v_{\hbar}(x)\right\} .
$$

From (5.9) and Lemma 5.4, it follows that $\lim _{\hbar \rightarrow 0} m_{\hbar}=0$. Thus, given $a>0$, let $\hbar_{0}>0$ be such that $m_{\hbar}<a / 2$, for all $\hbar \in\left(0, \hbar_{0}\right]$. Then, $\left(u_{\hbar}+v_{\hbar}-a\right)^{+}=0$ in $\partial A_{R_{1}}^{R_{2}}$. Since $u_{\hbar}+v_{\hbar}$ satisfies the equation

$$
-\hbar^{2} \Delta(u+v)+V(x)(u+v)=f(x, u)+g(x, v) \text { in } \mathbb{R}^{N},
$$


we obtain

$$
\begin{aligned}
\int_{D} \hbar^{2}\left|\nabla\left(u_{\hbar}+v_{\hbar}-a\right)^{+}\right|^{2} d x= & \int_{D}\left[-V(x) u_{\hbar}+f\left(x, u_{\hbar}\right)\right]\left(u_{\hbar}+v_{\hbar}-a\right)^{+} d x \\
& +\int_{D}\left[-V(x) v_{\hbar}+g\left(x, v_{\hbar}\right)\right]\left(u_{\hbar}+v_{\hbar}-a\right)^{+} d x,
\end{aligned}
$$

where $D=\mathbb{R}^{N} \backslash \overline{A_{R_{1}}^{R_{2}}}$. By (2.6), we have

$$
-V(x) s+f(x, s) \leq 0 \quad \text { and } \quad-V(x) s+g(x, s) \leq 0,
$$

for all $x \in \mathbb{R}^{N} \backslash A_{R_{1}}^{R_{2}}$ and $s \geq 0$. Thus,

$$
\int_{D} \hbar^{2}\left|\nabla\left(u_{\hbar}+v_{\hbar}-a\right)^{+}\right|^{2} d x \leq 0 .
$$

Hence, $u_{\hbar}+v_{\hbar}-a \leq 0$ in $\mathbb{R}^{N} \backslash \overline{A_{R_{1}}^{R_{2}}}$. Now, choosing $a=\min \left\{a_{1}, a_{2}\right\}$, where $a_{1}$ and $a_{2}$ were defined in (2.3), we conclude that $u_{\hbar} \leq a_{1}$ and $v_{\hbar} \leq a_{2}$ in $\mathbb{R}^{N} \backslash \overline{A_{R_{1}}^{R_{2}}}$. Then,

$$
f\left(x, u_{\hbar}\right)=f\left(u_{\hbar}\right) \quad \text { and } \quad g\left(x, v_{\hbar}\right)=g\left(v_{\hbar}\right) \quad \text { in } \mathbb{R}^{N} \backslash \overline{A_{R_{1}}^{R_{2}}} .
$$

Therefore, $\left(u_{\hbar}, v_{\hbar}\right)$ is a solution of $(1.1)$ for all $\hbar \in\left(0, \hbar_{0}\right]$.

Proof of Theorem 1.3. The proof is analogous to the proof of Theorem 1.2 with Lemma 5.4 replaced by Lemma 5.5 .

\section{REFERENCES}

[1] C.O. ALves, Existence of positive solutions for an equation involving supercritical exponent in $\mathbb{R}^{N}$, Nonlinear Anal. 42 (2000), 573-581.

[2] C.O. Alves, J.M. Do Ó And M.A.S. Souto, Local mountain-pass for a class of elliptic problems in $\mathbb{R}^{N}$ involving critical growth, Nonlinear Anal. 46 (2001), 495-510.

[3] A. Ávila AND J. YANG, On the existence and shape of least energy solutions for some elliptic systems, J. Differential Equations 191 (2003), 348-376.

[4] D. Bonheure, E.M. Dos Santos And M. Ramos, Ground state and non-ground state solutions of some strongly coupled elliptic systems, Trans. Amer. Math. Soc. 364 (2012), $447-491$.

[5] H. Berestycki And P.L. Lions, Nonlinear scalar field equations - I and II, Arch. Ration. Mech. Anal. 82 (1983), 313-376.

[6] D. Bonheure and M. Ramos, Multiple critical points of perturbed symmetric strongly indefinite functionals, Ann. Inst. H. Poincaré Anal. Non Linéaire 26 (2009), 675-688.

[7] J. Byeon And L. JeAnjeAn, Standing waves for nonlinear Schrödinger equations with a general nonlinearity, Arch. Ration. Mech. Anal. 185 (2007), 185-200.

[8] J. ByeOn AND Z. WANG, Standing waves with a critical frequency for nonlinear Schrdinger equations, Arch. Ration. Mech. Anal. 165 (2002), 295-316.

[9] Ph. Clément, D. G. De Figueiredo and E. Mitidieri, Positive solutions of semilinear elliptic systems, Comm. Partial Differential Equations 17 (1992), 923-940.

[10] D.G. De Figueiredo, Nonlinear elliptic systems. An. Acad. Brasil. Ciênc. 72 (2000), 453-469. 
[11] _ Semilinear elliptic systems: existence, multiplicity, symmetry of solutions, Handbook of differential equations, Vol. 5: Stationary Partial Differential Equations (M. Chipot, ed.), Elsevier, 2008, 1-48.

[12] D.G. De Figueiredo And J. YAnG, Decay, symmetry and existence of solutions of semilinear elliptic systems, Nonlinear Anal. 33 (1998), 211-234.

[13] M. Del Pino AND P.L. Felmer, Local mountain-pass for semilinear elliptic problems in unbounded domains, Calc. Var. Partial Differential Equations 4 (1996), 121-137.

[14] J. Hulshof, R.C.A.M. VAn DER Vorst, Differential systems with strongly indefinite variational structure, J. Funct. Anal. 114 (1993), 32-58.

[15] T.C. LIN AND J. WEI, Spikes in two-component systems of nonlinear Schrödinger equations with trapping potentials, J. Differential Equations 229 (2006), 538-569.

[16] O.H. MiYAGAKI, On a class of semilinear elliptic problems in $\mathbb{R}^{N}$ with critical growth, Nonlinear Anal. 29 (1997), 773-781.

[17] A. MoAmeni, Existence of soliton solutions for a quasilinear Schrödinger equation involving critical exponent in $\mathbb{R}^{N}$, J. Differential Equations 229 (2006), 570-587.

[18] J.D. Murray, Mathematical biology I, Spatial models and biomedical applications, third edition. Interdisciplinary Applied Mathematics, Vol. 18, Springer-Verlag, New York, 2003. xxvi+811 pp.

[19] P.H. Rabinowitz, On a class of nonlinear Schrödinger equations, Z. Angew Math. Phys. 43 (1992), 272-291.

[20] M. RAmos AND H. TAVARES, Solutions with multiple spike patterns for an elliptic system, Calc. Var. Partial Differential Equations 31 (2008), 1-25.

[21] M. Ramos AND J. YANG, Spike-layered solutions for an elliptic system with Neumann boundary conditions, Trans. Amer. Math. Soc. 357 (2005), 3265-3284.

[22] E. SchröDinger, Collected Papers on Wave Mechanics, Third Edition, AMS Chelsea Publishing, 2003.

[23] B. Sirakov, On the existence of solutions of Hamiltonian elliptic systems in $\mathbb{R}^{N}$, Adv. Differential Equations 5 (2000), 1445-1464.

[24] Standing wave solutions of the nonlinear Schrödinger equation in $\mathbb{R}^{N}$, Ann. Mat. Pura Appl. 181 (2002), 73-83.

[25] B. Sirakov And S.H.M. SoAres, Soliton solutions to systems of coupled Schrödinger equations of Hamiltonian type, Trans. Amer. Math. Soc. 362 (2010), 5729-5744.

[26] W.A. Strauss, Existence of solitary waves in higher dimensions, Comm. Math. Phys. 55 (1977), 149-162.

José A. CArdoso

Department of Mathematics

Federal University of Sergipe

49000-100 São Cristovão, SE, BRAZIL

E-mail address: andersonjvc@ufs.br

JoÃo M. DO Ó AND Everaldo Medeiros

Department of Mathematics

Federal University of Paraíba

58059-900 João Pessoa, PB, BRAZIL

E-mail address: jmbo@pq.cnpq.br, everaldo@mat.ufpb.br

TMNA : Volume $47-2016-\mathrm{N}^{\mathrm{O}} 2$ 